\title{
The relative influences of product volume, delivery format and alcohol concentration on dry-time and efficacy of alcohol-based hand rubs
}

\author{
David R Macinga ${ }^{1,2^{*}}$, David J Shumaker ${ }^{1}$, Heinz-Peter Werner ${ }^{3}$, Sarah L Edmonds ${ }^{1}$, Rachel A Leslie , Albert E Parker ${ }^{4,5}$ \\ and James W Arbogast ${ }^{1}$
}

\begin{abstract}
Background: Alcohol-based hand rubs (ABHR) range in alcohol concentration from 60-95\% and are available in a variety of delivery formats, such as rinses, gels, and foams. Recent studies suggest that some ABHR foams dry too slowly, thereby encouraging the use of inadequate volumes. This study investigates the influence of product volume, delivery format, and alcohol concentration on dry-time and antimicrobial efficacy of ABHR foams, gels and rinses.

Methods: ABHR dry-times were measured using volunteers to determine the influences of product volume, delivery format, and alcohol concentration. ABHR efficacies were evaluated according to the European Standard for Hygienic Hand Disinfection (EN 1500) using 3-mL application volumes rubbed for $30 \mathrm{~s}$, and additionally, using volumes of the products determined to rub dry in $30 \mathrm{~s}$.

Results: Volumes of six ABHR determined to rub dry in $30 \mathrm{~s}$ ranged from $1.7 \mathrm{~mL}$ to $2.1 \mathrm{~mL}$, and the rate of drying varied significantly between products. ABHR dry-times increased linearly with application volume and decreased linearly with increasing alcohol concentration, but were not significantly influenced by product format. An ABHR foam $(70 \% \mathrm{EtOH})$, rinse $(80 \% \mathrm{EtOH})$, and gel $(90 \% \mathrm{EtOH})$ each met EN 1500 efficacy requirements when tested at a volume of $3 \mathrm{~mL}$, but failed when tested at volumes that dried in $30 \mathrm{~s}$.

Conclusions: Application volume is the primary driver of ABHR dry-time and efficacy, whereas delivery format does not significantly influence either. Although products with greater alcohol concentration dry more quickly, volumes required to meet EN 1500 can take longer than $30 \mathrm{~s}$ to dry, even when alcohol concentration is as high as $90 \%$. Future studies are needed to better understand application volumes actually used by healthcare workers in practice, and to understand the clinical efficacy of ABHR at such volumes.
\end{abstract}

Keywords: Alcohol-based hand rub, ABHR foam, Application volume, Dry-time, In vivo efficacy, EN 1500

\section{Background}

Alcohol-based hand rubs (ABHRs) are recommended for routine hand disinfection in healthcare settings when hands are not visibly soiled [1,2]. Their role and importance has been highlighted by numerous studies associating their use with clinical reductions in hospital-acquired infections (HAIs) [1,2]. ABHR are available in a number of different delivery formats, such as rinses (i.e., thin liquids), gels, and

\footnotetext{
* Correspondence: macingad@gojo.com

'GOJO Industries, Inc, One GOJO Plaza, Suite 500, Akron, OH 44311, USA ${ }^{2}$ Department of Integrative Medical Sciences, Northeastern Ohio Medical University, Rootstown, Ohio 44272, USA

Full list of author information is available at the end of the article
}

foams. Each product format may offer a unique functional benefit, depending on the situation. For example, because gels and foams tend to drip from the hands less than rinses, the entire volume is delivered to the hands. And, beyond their functional attributes, there can be aesthetic preferences for specific ABHR formats [3].

Despite a large body of evidence supporting the use of ABHR for infection prevention, consensus is lacking as to the appropriate amount of a product that should be applied to the hands. The US Centers for Disease Control and Prevention $(C D C)$ Guidelines for Hand Hygiene in Healthcare Settings acknowledges that the ideal volume of product to apply to the hands is unknown, but states that, 
if hands dry before 10 to $15 \mathrm{~s}$, an insufficient amount was used [1]. The World Health Organization (WHO) Guidelines on Hand Hygiene in Health Care recommends that a "palmful" of product be used and states that the hand-hygiene event should take 20-30 s [2]. Despite lack of specific guidance on application volume, it has been clearly demonstrated by in-vivo microbiological studies and in clinical settings that antimicrobial efficacy of ABHR is directly proportional to application volume, and that ABHR will not meet global efficacy standards if insufficient volumes of product are applied [4-7]. It should be noted, further, that success criteria for current efficacy standards are arbitrary and not based on clinical outcomes $[8,9]$.

A recent study by Kampf et al. found that recommended use volumes for ABHR foams containing 62\% ethanol exceeded the WHO recommended dry-time of $30 \mathrm{~s}$, and that a volume that rubbed dry in $30 \mathrm{~s}(1.6 \mathrm{~mL})$ was insufficient to meet the efficacy criterion of EN 1500 [10]. The authors concluded that because the "...time required for dryness often exceeds the recommended $30 \mathrm{~s}$... only a small volume of these foams (below that required to meet efficacy norms) is likely to be applied in clinical practice". The study evaluated only ABHR foams, and all test products contained the same alcohol concentration; therefore, broad conclusions should not be drawn as to whether the observed drying rates are attributable to product format or to alcohol concentration. A study by Rotter et al. found that $3 \mathrm{~mL}$ of the EN 1500 Reference Product required, on average, more than $49 \mathrm{~s}$ to dry, despite the method specifying a rub-time of $30 \mathrm{~s}$ [11]. These data suggest that dry-time versus volume of an ABHR, regardless of delivery format, is an important relationship for study.

The objectives of this study were 1) to determine the influence of product volume, delivery format, and alcohol concentration on ABHR dry-time and antimicrobial efficacy, and 2) to investigate the efficacy of ABHRs when applied at volumes drying in $30 \mathrm{~s}$, as specified by WHO.

\section{Methods}

\section{Test Products}

The identities of the test products used in this study, listed in Table 1, were blinded to the test subjects. Product densities, measured using an Anton Paar DMA 4500 Density Meter at $60^{\circ} \mathrm{F}$, were used to convert ABHR mass to volume.

\section{Determination of ABHR dry-times}

ABHR gels, rinses, and ethanol-in-water solutions were applied using adjustable pipettes set to a desired volume. For gels, positive-displacement pipettes were used. ABHR foams were dispensed from pumps delivering a known constant quantity $(0.4 \mathrm{~mL})$, and total volumes were controlled by the number of pump activations. A specified quantity of an ABHR was placed in a subject's cupped palms, and the subject rubbed the product onto all surfaces of the hands up to the wrists "until the hands felt dry" to them. No instructions were given regarding rub-in technique. A calibrated digital timer was used to record the time interval from when a subject began rubbing to when they indicated their hands felt dry. After using each test product, subjects washed their hands with a bland soap and waited at least 30 minutes before a subsequent evaluation. Ethical approval was not considered necessary for this part of the study.

\section{Comparison of $\mathrm{ABHR}$ drying rates among multiple products} Dry-times for six ABHRs representing different product formats, and ranging in ethanol concentration from $62 \%$ to $90 \%$ (Table 2), were measured across a range of application volumes. A panel of thirteen subjects evaluated multiple volumes of the ABHR products in random order, a single product per test day. Mean dry-times recorded from all subjects were plotted against the product volumes used and analyzed by linear regression (GraphPad Prism 5.04, GraphPad Software, Inc., San Diego, CA) to determine the rate of drying $(\mathrm{s} / \mathrm{mL})$ and the volume of each product that should dry in $30 \mathrm{~s}$. Because variability of the dry-times tended to increase with increased volume (i.e., demonstrated heteroscedasticity), a weighted regression model with fixed effects for Product and the Product-Volume interaction was used to compare drytime rates as volume of product increased. To account for the repeated measures from each subject, the model also included a random intercept and rate for subject in the free statistical and graphing program $\mathrm{R}$ $[12,13]$. Residual and normal probability plots were used to confirm that heteroscedasticity of variance was adequately modeled. To maintain a family-wise false discovery rate of $5 \%$ among the follow-up $t$ tests, a Benjamini-Hochberg correction was applied.

\section{Relationship between ABHR dry-time and product delivery format}

To evaluate the specific influence of delivery format (gel, rinse, or foam) on dry-time, a panel of nurses evaluated each of three closely related test products (i.e., having identical ethanol concentrations and nearly identical excipient ingredient composition) delivered at two specific volumes, $0.8 \mathrm{~mL}$ and $1.6 \mathrm{~mL}$, estimated to dry in approximately $15 \mathrm{~s}$ and $30 \mathrm{~s}$, respectively, based on data for application volume versus dry-time. The sample size $(n=30)$ was calculated specifically to enable detection of a difference in dry-times as small as $5 \mathrm{~s}$ at $95 \%$ power. The calculation was based on data from the testing of dry-times versus application volume 
Table 1 Products tested in this study

\begin{tabular}{|c|c|c|c|c|}
\hline Code & Test product name & Manufacturer & Active ingredient & Density ( $\mathrm{g} / \mathrm{mL})$ \\
\hline Foam A & PURELL ${ }^{\oplus}$ Instant Hand Sanitizer Foam & GOJO Industries & $62 \%$ ethanol $(v / v)$ & 0.8940 \\
\hline Foam B & PURELL ${ }^{\circledR}$ Advanced Instant Hand Sanitizer Foam & GOJO Industries & $70 \%$ ethanol $(v / v)$ & 0.8739 \\
\hline Gel C & PURELL ${ }^{\oplus}$ Advanced Instant Hand Sanitizer & GOJO Industries & $70 \%$ ethanol $(v / v)$ & 0.8739 \\
\hline Rinse D & Experimental Prototype & GOJO Industries & $70 \%$ ethanol $(v / v)$ & 0.8739 \\
\hline Rinse $\mathrm{E}$ & WHO-recommended hand rub formulation with ethanol & $\mathrm{n} / \mathrm{a}$ & $80 \%$ ethanol (v/v) & 0.8661 \\
\hline \multirow[t]{2}{*}{ Gel F } & Sterillium ${ }^{\circledast}$ Comfort Ge ${ }^{\mathrm{Tm}}$ & Bode Chemie & $90 \%$ ethanol $(\mathrm{v} / \mathrm{v})^{\mathrm{a}}$ & 0.8331 \\
\hline & & & [85\% ethanol $(\mathrm{w} / \mathrm{w})]$ & \\
\hline
\end{tabular}

${ }^{a}$ Ethanol concentration on product label is reported as weight per weight $(\mathrm{w} / \mathrm{w})$; $(\mathrm{v} / \mathrm{v})$ concentration was determined analytically in the authors' laboratory (See Methods).

using thirteen subjects (Table 2). Rinse D represented the base formulation, with Foam B differing only by addition of a foaming agent $(<2 \% \mathrm{w} / \mathrm{w})$, and $\mathrm{Gel} \mathrm{C}$ by addition of a gelling agent $(<0.3 \% \mathrm{w} / \mathrm{w})$. Each formulation comprised more than $97 \%$ alcohol and water $w / w$, with non-volatile ingredients contributing less than $3 \%$ $\mathrm{w} / \mathrm{w}$. In addition to recording dry-times, occurrence of product dripping from a subject's hands during application and rubbing was noted. These data were analyzed using an ANOVA (and fit in R) that accounted for the repeated measures from each subject by including a random effect for subject and fixed effects, including the two-way interaction, for product format and dripping events. From this ANOVA, three Bonferroni simultaneous $90 \%$ confidence intervals (CIs) were generated to compare the mean dry-times amongst the three product formats, with statistical equivalence at $95 \%$ confidence concluded if all three Cls were contained in the interval $(-5,5)[14]$. In other words, mean differences as large as 5 seconds were assumed negligible and not of practical importance.

\section{Relationship between ABHR dry-time and ethanol concentration}

In a separate, but related experiment a panel of eleven subjects was used to determine dry-times for $1.7-\mathrm{mL}$ volumes of five ethanol-in-water rinse solutions ranging in concentration from $50 \%$ to $90 \% \mathrm{w} / \mathrm{w}$ in $10 \%$ increments. The 1.7-mL volume was selected based on data from the testing of dry-times versus application volume, viz., the volume of the two in-test ABHR foams that rubbed dry in $30 \mathrm{~s}$ (Table 2). A repeated-measures regression analysis with a random intercept and rate for subject was applied to determine the linear relationship between ethanol concentration and ABHR dry-time (in R). A repeated-measures ANOVA (Minitab 16) was used to compare the mean dry-times amongst the five ethanol concentrations used in the study, with a random effect for subject.

\section{In-vivo evaluation of antimicrobial efficacy}

The influence of application volume on ABHR efficacy was evaluated in two studies conducted according to the CEN phase 2/step 2 standard EN 1500 rev:2009 (Hygienic Handrub) methodology, as described previously $[8,15]$. Studies were conducted in Germany and were performed in compliance with the Declaration of Helsinki (DoH): Ethical Principles for Medical Research Involving Human Subjects (October 2013). Ethics board approval was not required based on the classification of Escherichia coli K12 (NCTC 10538) as a Risk Group 1 non-pathogenic organism by the German Safety Ordinance on Gene Technology. The laboratory was accredited in accordance with EN ISO 17025 (EN 45000) and recognized

Table 2 Statistical comparison of drying rates and volumes of six ABHR test products as a function of volume

\begin{tabular}{|c|c|c|c|c|c|c|c|c|}
\hline Test product & Active ingredient & Volume in $\mathrm{mL}$ drying in $30 \mathrm{~s}^{\mathrm{a}}$ & Drying rate in $\mathrm{s} / \mathrm{mL}(95 \% \mathrm{Cl})^{\mathrm{b}}$ & \multicolumn{5}{|c|}{ Drying rate Significance groups ${ }^{c}$} \\
\hline Foam A & $62 \%$ ethanol (v/v) & 1.7 & $18.2(15.5-21.0)$ & 1 & & & & \\
\hline Foam B & $70 \%$ ethanol $(\mathrm{v} / \mathrm{v})$ & 1.7 & $17.2(14.5-19.8)$ & 1 & 2 & & & \\
\hline Rinse D & $70 \%$ ethanol $(v / v)$ & 1.7 & $15.5(12.9-18.0)$ & & 2 & 3 & & \\
\hline Gel C & $70 \%$ ethanol $(v / v)$ & 1.9 & $14.0(11.5-16.4)$ & & & 3 & 4 & \\
\hline Rinse $\mathrm{E}$ & $80 \%$ ethanol $(v / v)$ & 2.0 & $12.8(10.4-15.2)$ & & & & 4 & 5 \\
\hline Gel F & $90 \%$ ethanol $(\mathrm{v} / \mathrm{v})^{\mathrm{d}}$ & 2.1 & $12.2(9.8-14.7)$ & & & & & 5 \\
\hline
\end{tabular}

${ }^{\mathrm{a}}$ Determined from the simple linear regression analysis where $\mathrm{T}=30 \mathrm{~s}$.

${ }^{\mathrm{b} C h a n g e}$ in dry-time per $\mathrm{mL}$ determined by a weighted regression model (see Methods).

${ }^{\mathrm{c}}$ Test products with the same number are not significantly different (See Methods).

${ }^{d}$ Concentration on product label is reported as weight per weight (w/w); (v/v) concentration was determined analytically in the authors' laboratory (See Methods). 
by ZLG, German Central Authority of the federal states for Health Protection of Pharmaceuticals and Medical Products. All areas of testing were approved and reported to the Ministry of Labour, Gender and Social Affairs of the Federal State of Mecklenburg Western Pomerania, Germany. Written informed consent was obtained from each subject prior to their participation in the study. A Latin-square design was used in each study to test three test products and a reference product $(60 \%$ isopropyl alcohol), with 20 subjects per study for a total of 40 subjects. The reference product was evaluated as specified in the standard: $3 \mathrm{~mL}$ rubbed for $30 \mathrm{~s}$, followed by an additional $3 \mathrm{~mL}$ rubbed for $30 \mathrm{~s}$. Test products were evaluated by each subject using a single application of each product. In the first study, test products were evaluated at an application volume of $3 \mathrm{~mL}$ and rubbed for a timed $30 \mathrm{~s}$, in accordance with EN 1500 rev:2009 procedure, after which the fingertips were rinsed for $5 \mathrm{~s}$ under running tap water, excess water shaken off, and hands immediately sampled. In the second study, the procedure was modified such that test products were rubbed until completely dry and then, without rinsing, sampled immediately thereafter. Neutraliser $(3.0 \%$ polysorbate $80+3.0 \%$ saponine $+0.1 \%$ histidine $+0.1 \%$ cysteine) validated prior to testing was used as the sampling fluid and diluent for all products tested. Application volumes were $1.6 \mathrm{~mL}$ of Foam B, $2.0 \mathrm{~mL}$ of Rinse E, and $2.1 \mathrm{~mL}$ of Gel F, volumes of each indicated to rub dry in $30 \mathrm{~s}$ based on testing described above (Table 2). Because the volume of Foam B indicated to dry in $30 \mathrm{~s}$ $(1.7 \mathrm{~mL})$ could not be achieved with the available pump device, the smaller, conservative volume was used. A $\log _{10}$ reduction factor (RF) produced by each product per subject was calculated, and the HodgesLehman test was applied for comparison of the mean $\log _{10}$ RF to the mean $\log _{10}$ RF for the reference procedure, as recommended by EN 1500 rev:2009. In addition, mean $\log _{10}$ RFs were compared between the test products using a repeated-measures ANOVA with a random effect for Day (Minitab 16). Follow-up Tukey 95\% CIs of the mean $\log _{10}$ RF difference between each test product and reference were generated. Test products that demonstrated inferiority (i.e., mean $\log _{10}$ RF significantly less than that observed from the reference solution) were classified as not meeting the norm. Non-inferiority of each test product compared to the reference was analyzed as specified by EN 1500 rev:2009; that is, non-inferiority was concluded if all of the Tukey 95\% CIs were contained in the interval $(-\infty, 0.6]$.

In all statistical tests of differences, a level of $5 \%$ (i.e., $P \leq 0.05)$ was used to determine statistical significance. The tests for equivalence amongst product formats were also performed at a significance level of $5 \%$, as explained above. However, to be consistent with EN 1500 rev:2009, the non-inferiority analyses were conducted at a significance level of $2.5 \%$.

\section{Results}

Influence of ABHR application volume on dry-time

Dry-times of six ABHR test products, representing different product formats and alcohol concentrations (Table 1), were evaluated at multiple application volumes. Figure 1 illustrates the results for a $70 \% \mathrm{v} / \mathrm{v}$ ethanol gel (Gel C). A significant correlation found between application volume and the time for each test product to dry supported calculation of a rate of drying $(\mathrm{s} / \mathrm{mL})$ and established the volume of each product that should dry in $30 \mathrm{~s}$ (Table 2). The volumes indicated to dry in $30 \mathrm{~s}$ ranged from $1.7 \mathrm{~mL}$ (Foam A, Foam B, Rinse D) to $2.1 \mathrm{~mL}$ (Gel F). There were significant differences in the rates of drying, with Foam A having the slowest drying rate $(18.2 \mathrm{~s} / \mathrm{mL})$, and Gel F having the fastest drying rate $(12.2 \mathrm{~s} / \mathrm{mL})$ (Table 2$)$. Because the test products differed in both alcohol concentration and product format, the influence of each variable on drytime was explored.

\section{Influence of product delivery format on ABHR dry-time}

To control for other variables, dry-times of three ABHR (Foam B, Gel C, and Rinse D) containing 70\% ethanol and nearly identical excipient ingredients were compared (Table 3). Application volumes of $1.6 \mathrm{~mL}$ and $0.8 \mathrm{~mL}$ were chosen to produce dry-times of approximately $30 \mathrm{~s}$

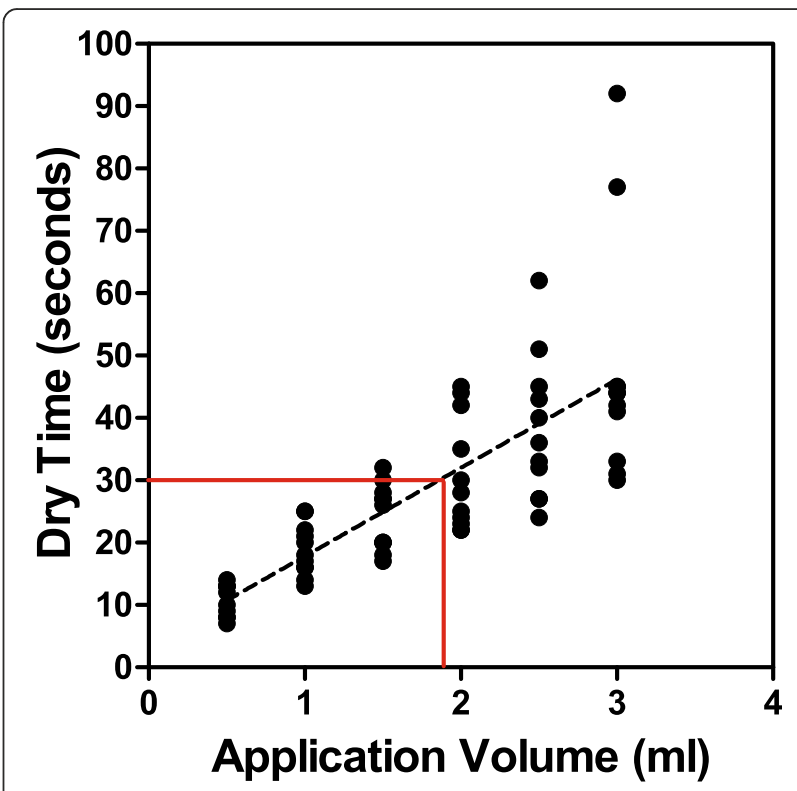

Figure 1 Relationship between ABHR application volume and dry-time. Dry-time plotted against ABHR application volume of Gel C. Black circles represent measured dry-times recorded from 11 volunteers at each application volume tested. The black dashed line is the best-fit linear regression, and the solid red line indicates the calculated volume that should rub dry in $30 \mathrm{~s}$. 
Table 3 Mean dry-times of three ABHR test products evaluated at two application volumes

\begin{tabular}{|c|c|c|c|c|c|c|}
\hline \multirow{3}{*}{ Test product } & \multicolumn{3}{|c|}{$0.8 \mathrm{~mL}$ application volume } & \multicolumn{3}{|c|}{$1.6 \mathrm{~mL}$ application volume } \\
\hline & Mean dry-time in $s^{a}$ & Range & \# of Subjects & Mean dry-time in $s^{a}$ & Range & \# of Subjects \\
\hline & $(95 \% \mathrm{Cl})$ & in $\mathrm{s}$ & dripping product & $(95 \% \mathrm{Cl})$ & In $\mathrm{s}$ & dripping product \\
\hline Foam B & $14.7(13.4-16.1)$ & $7-23$ & 0 & $28.3(26.0-30.7)$ & $18-49$ & 1 \\
\hline Gel C & $16.0(14.4-17.7)$ & $10-25$ & 0 & $25.0(22.8-27.2)$ & $14-40$ & 4 \\
\hline Rinse D & $17.0(15.4-18.7)$ & $9-25$ & 4 & $25.0(22.9-27.1)$ & $15-38$ & 13 \\
\hline
\end{tabular}

${ }^{\mathrm{a}} N=30$ test subjects; each evaluated all six test configurations in a randomized order.

and $15 \mathrm{~s}$, respectively, consistent with recommendations of $\mathrm{WHO}$ and $\mathrm{CDC}[1,2]$. At a $0.8-\mathrm{mL}$ volume, CIs of differences in mean dry-times (see Methods) show that differences in the mean dry-times amongst the three products were no greater than $3.5 \mathrm{~s}$. Thus, the three products yielded statistically equivalent dry-times, on the average. At the 1.6- $\mathrm{mL}$ application volume, dripping of Rinse D was observed more frequently (13 of 30 subjects) than of either Gel C (4 of 30 subjects) or Foam A (1 of 30 subjects). Taking product dripping into account, CIs show that differences in the mean dry-times amongst the three products were no greater than $4.9 \mathrm{~s}$. Again, drytimes of the three products were statistically equivalent, on the average.

\section{Relationship between ABHR dry-time and ethanol concentration}

Figure 2 illustrates that ABHR dry-time decreased linearly with increase in ethanol concentration, a relationship

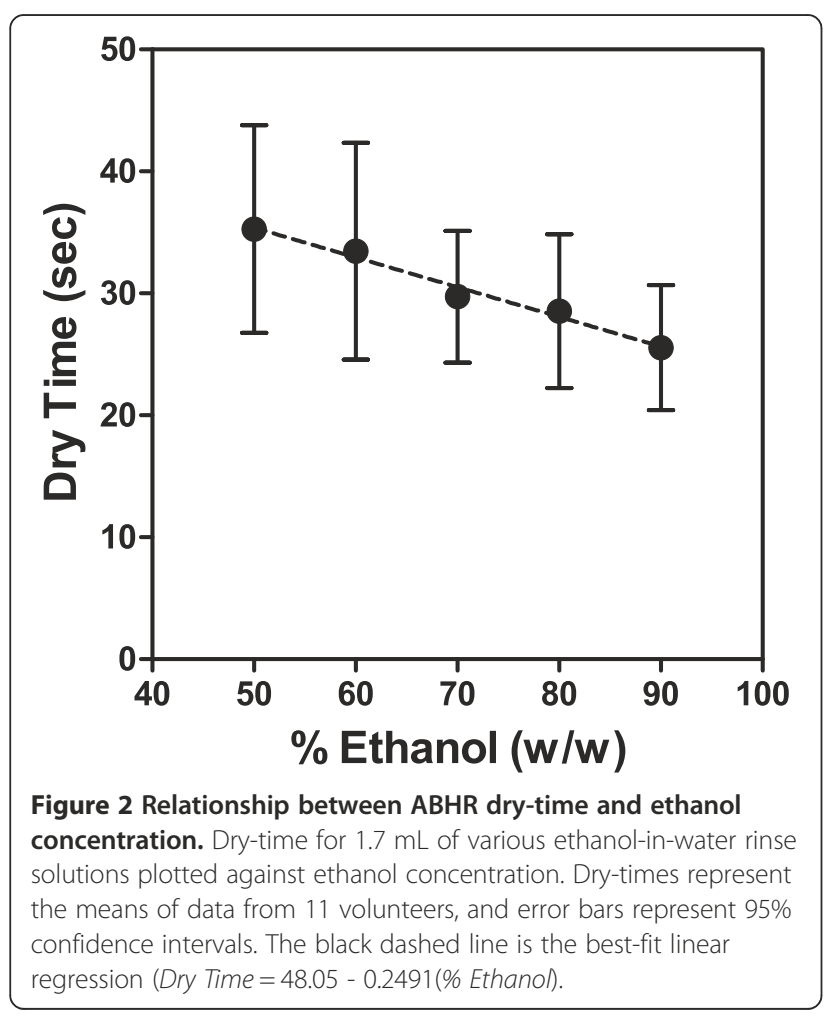

found to be statistically significant $(P=0.0035)$. Furthermore, the mean time to dry for $50 \%$ ethanol was significantly greater than that of $80 \%(P=0.0479)$ and $90 \%$ ethanol $(P=0.0015)$, as was mean dry-time of $60 \%$ ethanol versus that of $90 \%$ ethanol $(P=0.0071)$.

\section{Influence of ABHR application volume on antimicrobial efficacy}

The efficacies of representative ABHR foam (Foam B), rinse (Rinse E), and gel (Gel F) formulations were evaluated according to the European norm EN 1500 at a standard volume of $3 \mathrm{~mL}$ (rubbed for $30 \mathrm{~s}$ and rinsed) and, also, at the approximate volumes determined from Table 2 to rub dry in $30 \mathrm{~s}$. The test products, chosen to represent each of the different product formats, contained different alcohol levels and, thus, different volumes drying in $30 \mathrm{~s}$ ). When evaluated at a volume of $3 \mathrm{~mL}$ and rubbed for $30 \mathrm{~s}$, the upper bounds of CIs of mean $\log _{10}$ RF differences of each of the three products from the mean $\log _{10}$ $\mathrm{RF}$ of the reference procedure was less than 0.6 (Table 4). Thus, efficacies of the three test products used at volumes of $3 \mathrm{~mL}$ were statistically non-inferior to efficacy of the reference product, thereby satisfying the requirements of EN 1500. Furthermore, efficacies of Foam A, Rinse E, and Gel $F$ were not significantly different from each other when evaluated at $3-\mathrm{mL}$ volumes $(P=0.686)$ or at volumes drying in $30 \mathrm{~s}(P \geq 0.4292)$. However, at volumes drying in $30 \mathrm{~s}$, the mean $\log _{10}$ RFs produced by the three ABHR were significantly inferior to that of the reference procedure, and thus failed to meet EN 1500 efficacy requirements.

\section{Discussion}

In this study, we evaluated the influence of several variables on ABHR dry-time, including product format, application volume, and alcohol concentration. Consistent with previous studies, ABHR dry-times increased proportionally to product application volume (Figure 1) $[10,11]$, and the rates at which different ABHR products dried varied significantly (Table 2). The volume of the two foams evaluated in this study drying in $30 \mathrm{~s}$ was $1.7 \mathrm{~mL}(1.5 \mathrm{~g})$, which is similar to that reported by Kampf et al. for ABHR foams (1.6 g) [10]. Furthermore, the drying rates calculated in Table 2 revealed that the two 
Table 4 Efficacy of three ABHRs evaluated according to procedures of EN 1500

\begin{tabular}{|c|c|c|c|c|c|c|c|c|}
\hline \multirow[t]{2}{*}{ Test ABHR } & \multicolumn{4}{|c|}{ Efficacy at 3-mL application volume } & \multicolumn{4}{|c|}{ Efficacy at volume drying in $30 \mathrm{~s}$} \\
\hline & $\begin{array}{l}\text { Application } \\
\text { volume }\end{array}$ & $\begin{array}{c}\text { mean log } \\
R F \pm S D\end{array}$ & $P$-value ${ }^{a}$ & $\mathrm{Cl}^{\mathrm{b}}$ & Application volume & $\begin{array}{c}\text { mean log } \\
R F \pm S D\end{array}$ & $P$-value ${ }^{a}$ & $\mathrm{Cl}^{\mathrm{b}}$ \\
\hline EN 1500 reference & $2 \times 3 \mathrm{~mL}^{\mathrm{c}}$ & $4.63 \pm 0.60$ & & & $2 \times 3 \mathrm{~mL}$ & $4.83 \pm 0.79$ & & \\
\hline Foam B & $3 \mathrm{~mL}$ & $4.56 \pm 0.68$ & 0.9299 & $(-0.23,0.37)$ & $1.6 \mathrm{~mL}^{\mathrm{d}}$ & $3.81 \pm 0.61$ & $<0.00005$ & $(0.65,1.38)$ \\
\hline Rinse $\mathrm{E}$ & $3 \mathrm{~mL}$ & $4.50 \pm 0.90$ & 0.6793 & $(-0.17,0.43)$ & $2.0 \mathrm{~mL}$ & $4.03 \pm 0.55$ & $<0.00005$ & $(0.441 .18)$ \\
\hline Gel F & $3 \mathrm{~mL}$ & $4.61 \pm 0.94$ & 0.9979 & $(-0.28,0.32)$ & $2.1 \mathrm{~mL}$ & $4.02 \pm 0.60$ & $<0.00005$ & $(0.44,1.17)$ \\
\hline
\end{tabular}

${ }^{\mathrm{a}}$ Comparison of each product with the reference.

${ }^{\mathrm{b}} 95 \%$ two-sided Cls on the mean $\log _{10} \mathrm{RF}$ for each product subtracted from the mean $\log _{10} \mathrm{RF}$ for the reference. By EN 1500 , if the upper confidence limit difference is less than 0.6 , then non-inferiority between the test and reference products is established.

${ }^{c}$ Reference product rubbed for a total of $60 \mathrm{~s}$, followed by a 5 -s rinse under running tap water.

${ }^{\mathrm{d}}$ The volume calculated to dry in $30 \mathrm{~s}$ was $1.7 \mathrm{~mL}$. This volume could not be achieved with available pump devices, so a lower (conservative) volume was used.

foams were the slowest drying products tested. However, careful examination of Table 2 in the context of alcohol concentration of each test product indicates that rate of drying was faster for products with higher alcohol concentration, regardless of format. Noting the inverse relationship between ABHR dry-time and alcohol concentration clearly demonstrated in Figure 2, the observed differences in test product drying rates likely result from differences in alcohol concentration. That is, as the alcohol concentration of ABHR is increased, the volume of product that will rub dry in $30 \mathrm{~s}$ also increases. Note that $2.1 \mathrm{~mL}$ of Gel F $(90 \% \mathrm{v} / \mathrm{v})$ dried in $30 \mathrm{~s}$, as did $1.7 \mathrm{~mL}$ of Gel A (62\% ethanol $\mathrm{v} / \mathrm{v}$ ), a difference of $0.4 \mathrm{~mL}$ (Table 2).

By evaluating products of equivalent alcohol concentration, differing only by the presence of thickening or foaming agents, delivery format was found to have no influence on ABHR dry-time (Table 3). This result is not surprising, considering that $\mathrm{ABHR}$ formulations consist predominantly of alcohol and water. Despite differences in physical characteristics, Foam B, Gel C, and Rinse D each comprise greater than $97 \%$ alcohol and water by weight and differ in composition by less than 3\%. This similarity is further evidenced by the identical densities of the 3 products (Table 1). Based on demonstrated influence of alcohol concentration on dry-time (Figure 2) and lack of influence of delivery format on dry-time, we would attribute the differences in drying rates to alcohol concentration.

In agreement with previous studies, product application volume was found to be a critical determinant of ABHR efficacy [4,7]. Each of the three products evaluated (Foam $\mathrm{B}$, Rinse E, and Gel F) met the performance criteria of EN 1500 when tested at 3-mL application volumes, but failed when tested at volumes drying in $30 \mathrm{~s}$ (Table 4). Statistical equivalence of the mean $\log _{10}$ RFs was established amongst the three products when applied in volumes that dried in $30 \mathrm{~s}$, despite the fact that Gel F, the test product with the highest alcohol concentration, was also tested at the largest application volume. Taken together, these data suggest that time that product remains wet on the hands may be the most important determinant of ABHR efficacy when evaluated under actual use conditions (i.e., rubbed until dry), irrespective of other variables.

By expanding the studies of Kampf et al. [10] to include gels and rinses we can expand upon their conclusions. Regardless of delivery format or alcohol concentration, the volume ABHR drying in $30 \mathrm{~s}$ is considerably less than $3 \mathrm{~mL}$. Furthermore, when $3 \mathrm{~mL}$ of product are used, drytimes are considerably greater than 30 s. Importantly, when used at volumes drying in $30 \mathrm{~s}$, gel, foam, and rinse products did not meet the efficacy criteria of EN 1500. Therefore, the assertion that the use of ABHR foams would encourage users to apply volumes too low to meet EN 1500 standards [10] should be expanded to include gels and rinses. The data presented herein suggest that it may be more appropriate to make ABHR application recommendations based on formulation specific efficacy data.

Indeed, there is currently limited understanding of the actual ABHR application volumes used by healthcare workers in practice, despite recommendations [1,2]. A recent survey of ABHR dispensers in U.S. hospitals found that outputs ranged from $0.6 \mathrm{~mL}$ to $1.3 \mathrm{~mL}$, and that product dry-times ranged from $12 \mathrm{~s}$ to $26 \mathrm{~s}$ [16]. Whether healthcare workers use more than one dispenser actuation is unknown; anecdotally, it is expected they most frequently do not. An often-cited publication by Voss and Widmer argued that the use of ABHR saves significant time and can promote hand hygiene compliance [17]. Note, however, that the time estimated for application of an ABHR was $20 \mathrm{~s}$; considerably shorter than the dry-time for the 3-mL volume required to meet $\mathrm{EN}$ 1500 efficacy requirements.

To better understand ABHR efficacy under clinical use conditions, "in particular, short application times and volumes", experts have called for the development of improved in-vivo protocols that more closely simulate real-world conditions [2,18]. EN 1500 specifies that test subjects rub $3 \mathrm{~mL}$ of test product onto the hands for 
$30 \mathrm{~s}$ and then to neutralize the remaining alcohol [8]. However, as Table 2 indicates, typical volumes of ABHRs drying in $30 \mathrm{~s}$ range from 1.7 to $2.1 \mathrm{~mL}$, and Figure 1 illustrates that $3 \mathrm{~mL}$ can take as long as $90 \mathrm{~s}$ to dry on the hands. This uncoupling of dry-time from application volume does not represent real use conditions. The modification to EN 1500 used in this study, where the test product is rubbed until dry, with no subsequent rinse, more closely simulates product usage in clinical settings and results in more accurate recommendations for application volume.

Our study has several limitations. For product dry-time experiments, that subjects did not use a standardized rubin technique may have had a minor impact on dry-times. However, adherence of healthcare workers to the standardized technique is unknown, but unlikely, and this technique has been previously shown to have minimal impact on ABHR efficacy [19]. Efficacy studies were performed using a limited number of subjects and a laboratory-based method with success criteria not linked to clinical outcome, and they should be understood in that way. Lastly, this study evaluated efficacy of only a single representative of each product delivery type (rinse, gel, and foam). Because product formulation also impacts efficacy [15], broad conclusions regarding the efficacy of other products should be made with caution.

\section{Conclusion}

In conclusion, application volume was found to be the primary driver of ABHR dry-time and, therefore, of efficacy. Our data suggest that ABHR application volumes consistent with WHO recommendations may fail to meet EN 1500 efficacy requirements, irrespective of delivery format or alcohol concentration. The implications of this are unknown, but should be of considerable concern. Further research is needed to better understand ABHR application volumes actually used by healthcare workers in clinical practice and how these relate to product effectiveness. Finally, there is a need to develop improved standard test methods that more closely reflect clinical use conditions and provide success criteria linked to clinical outcomes.

\section{Competing interests}

DRM, DJS, SLE, RAL and JWA are employed by GOJO, Industries, Inc., Akron, $\mathrm{OH}$, USA, a manufacturer of hand hygiene products including alcohol-based handrubs.

\section{Authors' contributions}

DRM, DJS, SLE, RAL, and JWA made substantial contributions to conception and design. DJS conducted all dry-time experiments. HPW was responsible for the EN 1500 phase 2/step 2 testing with volunteers. DRM, SLE, and RAL made substantial contributions to acquisition, analysis, and interpretation of data. AEP conducted all statistical analysis and helped with design of dry-time experiments. DRM drafted the manuscript, and all authors read and approved the final manuscript.

\section{Acknowledgements}

The authors thank Dr. John Mitchell for his critical review of this manuscript.

\section{Author details}

${ }^{1}$ GOJO Industries, Inc, One GOJO Plaza, Suite 500, Akron, OH 44311, USA. ${ }^{2}$ Department of Integrative Medical Sciences, Northeastern Ohio Medical University, Rootstown, Ohio 44272, USA. ${ }^{3}$ HygCen International GmbH, Werksgelände 24, Bischofshofen 5500, Austria. ${ }^{4}$ Center for Biofilm Engineering at Montana State University, Bozeman, Montana 59717, USA. ${ }^{5}$ Department of Mathematical Sciences at Montana State University, Bozeman, Montana 59717, USA.

Received: 17 January 2014 Accepted: 18 September 2014 Published: 20 September 2014

\section{References}

1. Boyce JM, Pittet D: Guideline for hand hygiene in health-care settings. Recommendations of the Healthcare Infection Control Practices Advisory Committee and the HICPAC/SHEA/APIC/IDSA Hand Hygiene Task Force. Society for Healthcare Epidemiology of America/Association for Professionals in Infection Control/Infectious Diseases Society of America. MMWR Recomm Rep 2002, 51:1-45. quiz.

2. World Health Organization: WHO Guidelines on Hand Hygiene in Health Care. Geneva: World Health Organization; 2009.

3. Traore O, Hugonnet S, Lubbe J, Griffiths W, Pittet D: Liquid versus gel handrub formulation: a prospective intervention study. Crit Care 2007, 11:R52.

4. Goroncy-Bermes P, Koburger T, Meyer B: Impact of the amount of hand rub applied in hygienic hand disinfection on the reduction of microbial counts on hands. J Hosp Infect 2010, 74:212-218.

5. Kampf G: How effective are hand antiseptics for the postcontamination treatment of hands when used as recommended? Am J Infect Control 2008, 36:356-360.

6. Larson EL, Eke PI, Wilder MP, Laughon BE: Quantity of soap as a variable in handwashing. Infect Control 1987, 8:371-375.

7. Macinga DR, Beausoleil CM, Campbell E, Mulberry G, Brady A, Edmonds SL, Arbogast JW: Quest for a realistic in vivo test method for antimicrobial hand-rub: introduction of a low-volume hand contamination procedure. Appl Environ Microbiol 2011, 77:8588-8594.

8. Comité Européen de Normalisation: EN 1500: Chemical disinfectants and antiseptics. Hygienic hand disinfection. Test method and requirement (phase 2, step 2). Brussels: Comité Européen de Normalisation; 1997.

9. Food and Drug Administration: Tentative final monograph for healthcare antiseptic drug products; proposed rule. Federal Register 1994, 59:31441-31450

10. Kampf G, Marschall S, Eggerstedt S, Ostermeyer C: Efficacy of ethanol-based hand foams using clinically relevant amounts: a cross-over controlled study among healthy volunteers. BMC Infect Dis 2010, 10:78

11. Rotter M, Suchomel M, Weinlich M, Kundi M: Impact of shortening the duration of application and the standardized rubbing sequence as well as the reduction of the disinfectant volume used for the hygienic hand rub with 2-propanol (60\% V/V) [Translation]. Hyg Med 2009, 34:19-23.

12. Pinhiero J, Bates D, DebRoy SSD, The R Development Core Team: nlme: Linear and nonlinear mixed effects models. in [R package version 3.1-104]. 2011.

13. R Development Core Team: $R$; A language and environment for statistical computing. Vienna, Austria: R Foundations for Statistical Computing; 2010.

14. Richter SJ, Richter C: A method for determining equivalence in industrial applications. Quality Engineering 2013, 14:375-380.

15. Edmonds SL, Macinga DR, Mays-Suko P, Duley C, Rutter J, Jarvis WR, Arbogast JW: Comparative efficacy of commercially available alcohol-based hand rubs and World Health Organization-recommended hand rubs: formulation matters. Am J Infect Control 2012, 40:521-525

16. Macinga DR, Edmonds SL, Campbell E, Shumaker DJ, Arbogast JW: Efficacy of novel alcohol-based hand rub products at typical in-use volumes. Infect Control Hosp Epidemiol 2013, 34:299-301.

17. Voss A, Widmer AF: No time for handwashing? Handwashing versus alcoholic rub: can we afford 100\% compliance? Infect Control Hosp Epidemiol 1997, 18:205-208. 
18. Rotter M, Sattar S, Dharan S, Allegranzi B, Mathai E, Pittet D: Methods to evaluate the microbicidal activities of hand-rub and hand-wash agents. J Hosp Infect 2009, 73:191-199.

19. Kampf G, Reichel M, Feil Y, Eggerstedt S, Kaulfers PM: Influence of rub-in technique on required application time and hand coverage in hygienic hand disinfection. BMC Infect Dis 2008, 8:149.

doi:10.1186/1471-2334-14-511

Cite this article as: Macinga et al:: The relative influences of product volume, delivery format and alcohol concentration on dry-time and efficacy of alcohol-based hand rubs. BMC Infectious Diseases 2014 14:511.

\section{Submit your next manuscript to BioMed Central and take full advantage of:}

- Convenient online submission

- Thorough peer review

- No space constraints or color figure charges

- Immediate publication on acceptance

- Inclusion in PubMed, CAS, Scopus and Google Scholar

- Research which is freely available for redistribution 